Annales Geophysicae (2003) 21: 863-867 (C) European Geosciences Union 2003

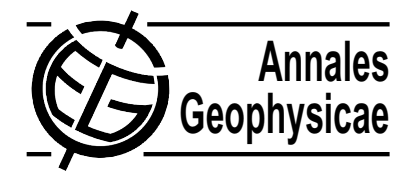

\title{
Reconstructing the long-term cosmic ray intensity: linear relations do not work
}

\author{
K. Mursula ${ }^{1}$, I. G. Usoskin ${ }^{2}$, and G. A. Kovaltsov ${ }^{3}$ \\ ${ }^{1}$ Department of Physical Sciences, P.O.B. 3000, FIN-90014, University of Oulu, Finland \\ ${ }^{2}$ Sodankylä Geophysical Observatory (Oulu unit), P.O.B. 3000, FIN-90014, University of Oulu, Finland \\ ${ }^{3}$ Ioffe Physical-Technical Institute, RU-194021, St. Petersburg, Russia
}

Received: 21 February 2002 - Revised: 3 October 2002 - Accepted: 18 October 2002

\begin{abstract}
It was recently suggested (Lockwood, 2001) that the cosmic ray intensity in the neutron monitor energy range is linearly related to the coronal source flux, and can be reconstructed for the last 130 years using the long-term coronal flux estimated earlier. Moreover, Lockwood (2001) reconstructed the coronal flux for the last 500 years using a similar linear relation between the flux and the concentration of cosmogenic ${ }^{10} \mathrm{Be}$ isotopes in polar ice. Here we show that the applied linear relations are oversimplified and lead to unphysical results on long time scales. In particular, the cosmic ray intensity reconstructed by Lockwood (2001) for the last 130 years has a steep trend which is considerably larger than the trend estimated from observations during the last 65 years. Accordingly, the reconstructed cosmic ray intensity reaches or even exceeds the local interstellar cosmic ray flux around 1900. We argue that these unphysical results obtained when using linear relations are due to the oversimplified approach which does not take into account the complex and essentially nonlinear nature of long-term cosmic ray modulation in the heliosphere. We also compare the long-term cosmic ray intensity based on a linear treatment with the reconstruction based on a recent physical model which predicts a considerably lower cosmic ray intensity around 1900.
\end{abstract}

Key words. Interplanetary physics (cosmic rays; heliopause and solar wind termination) - Geomagnetism and paleomagnetism (time variations, secular and long-term)

\section{Introduction}

Recently, Lockwood et al. (1999) and Lockwood (2001) (to be called here L01) estimated the coronal source flux $F_{S}$ for the time after 1868 using the geomagnetic $a a$ index. L01 also reconstructed the intensity of cosmic rays (CR) during that period using a simple linear relation between $F_{S}$ and cosmic rays (as measured by the Climax neutron monitor with a geomagnetic cutoff rigidity of about $3 \mathrm{GV}$ ). First, they calcu-

Correspondence to: K. Mursula (Kalevi.Mursula@oulu.fi) lated the linear relation between $F_{S}$ and CR intensity for the neutron monitor (NM) era after 1951. Then, this linear relation was used to reconstruct the CR intensity for the longer period since 1868 , using the coronal flux estimated earlier.

Lockwood (2001) also assumed a linear relation to exist between $F_{S}$ and the concentration of cosmogenic ${ }^{10} \mathrm{Be}$ isotopes in polar ice. ${ }^{10} \mathrm{Be}$ concentration responds mainly to the flux of cosmic rays with an energy of several $\mathrm{GeV}$ (Masarik and Beer, 1999; McCracken and McDonald, 2001). Accordingly, it is a good proxy of cosmic rays of the NM energy range (Beer, 2000). Calculating the linear relation between $F_{S}$ and ${ }^{10} B e$ data for the period $1868-1985$, L01 then extrapolated this relation to the whole period covered by the ${ }^{10} \mathrm{Be}$ record, thus reconstructing the very long-term $F_{s}$ profile since 1423. This reconstructed $F_{S}$ was suggested in L01 to form a new index of solar activity for very early times. It is important to note that only linear relations between the different solar/heliospheric parameters were used in L01.

Recently, we have also reconstructed the CR intensity in the past as measured by NM and by ${ }^{10} \mathrm{Be}$ (Usoskin et al., 2002a, to be called here U02). In that work we used a physical model rather than empirical linear relations, leading to quite different results from L01. In this paper we discuss the two CR reconstructions and show that the linear relations that were assumed in L01 between $F_{s}$ and cosmic rays (or their proxies) are oversimplified and lead to unreliable results. In Sect. 2 we discuss the reconstruction of cosmic ray intensity used in L01 and in U02 for the last 130 years. In Sect. 3 we re-analyse the results obtained in L01 from the ${ }^{10} \mathrm{Be}$ series. Finally, in Sect. 4 we give our conclusions and final remarks.

\section{Cosmic ray reconstruction}

\subsection{Linear reconstruction}

L01 reconstructed the CR intensity in the past using a simple linear relation between the calculated coronal magnetic flux $F_{S}$ and the Climax NM count rate for the time after 1951, and 


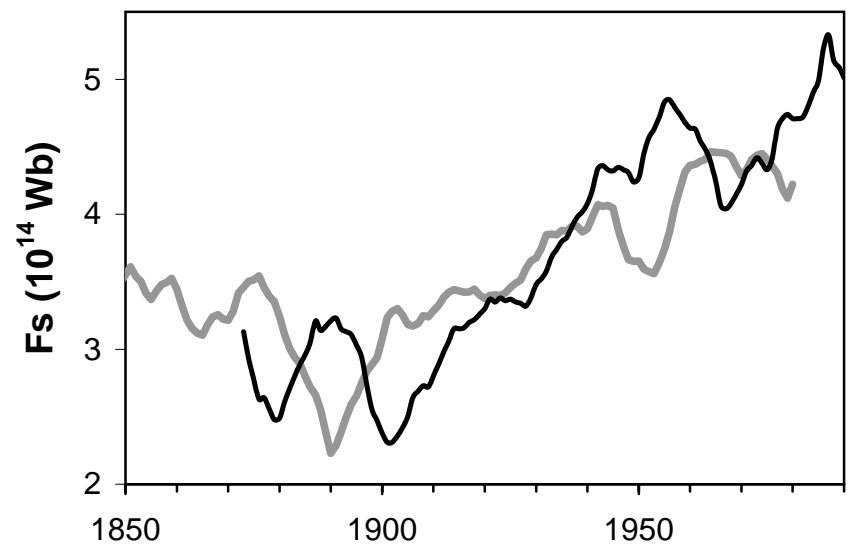

Fig. 1. The coronal source flux $F_{S}$ calculated by the method of Lockwood et al. (1999) from the geomagnetic aa series (black curve) and reconstructed from the ${ }^{10} \mathrm{Be}$ series (grey curve). The curves are 11-year running mean values.

then extrapolated it back in time. In order to repeat the reconstruction presented in L01, we have calculated the coronal source flux $F_{S}$ since 1868 from the $a a$ index using the recipe published by Lockwood et al. (1999). This $F_{S}$ series is depicted in Fig. 1. We note that it corresponds exactly to the coronal source flux calculated and shown in L01 (see Fig. 2a of L01). Similar to L01, we find the following linear regression between the annual values of Climax NM data CR(NM) and the calculated $F_{S}$ flux for the period of 1951-1999,

$C R(N M)=(5.25 \pm 0.11)-(0.28 \pm 0.025) \cdot F_{S}$,

where $\mathrm{CR}(\mathrm{NM})$ is given in counts $/ \mathrm{h} / 10^{5}$ and $F_{s}$ in $10^{14} \mathrm{~Wb}$. This coincides with the regression obtained in L01. Using this linear regression, we have calculated the CR(NM) series since 1868 from the $F_{s}$ series, similar to L01. (Note also that the geomagnetic cutoff rigidity has been assumed to remain constant when Eq. (1) is extrapolated over long time scales.) The reconstructed $\mathrm{CR}(\mathrm{NM})$ series is shown as a dotted line in Fig. 2, together with the $1 \sigma$ error. This series is also in a good agreement with the results presented in L01 (see, e.g. Fig. 10 of L01).

\subsection{Physical modeling}

In the recent CR reconstruction (U02) we used the following logical chain

$$
S A \stackrel{(1)}{\longrightarrow} F_{S} \stackrel{(2)}{\longrightarrow} \Phi \stackrel{(3)}{\longrightarrow} S \stackrel{(4)}{\longrightarrow} C R(N M) .
$$

So first, using the model by Solanki et al. (2000, 2002), we calculate the coronal source magnetic flux $F_{S}$ from sunspot activity SA (step 1 in Eq. 2). In step 2 we use the power-law relation between $F_{S}$ and the modulation strength $\Phi$ (Gleeson and Axford, 1968) derived by Usoskin et al. (2002a). Next, using our stochastic simulation model of CR transport in the heliosphere (Usoskin et al., 2002b), we calculate the modulated CR spectra $S$ at the Earth's vicinity (step 3). Finally, we calculate the NM count rate as an integral of a product of

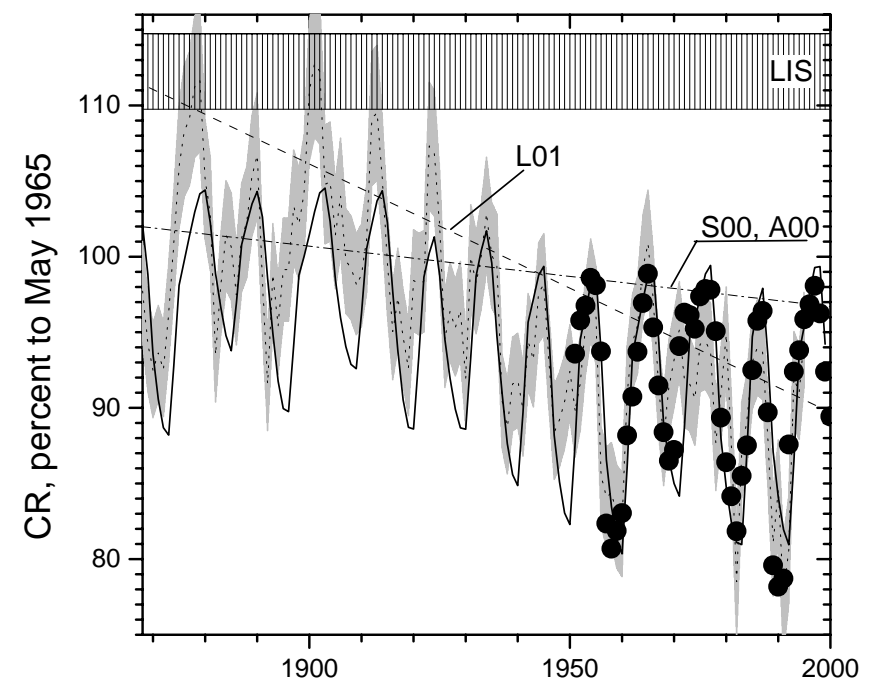

Fig. 2. Climax NM count rates (in percent to May 1965). Dotted curve with grey shading denotes the count rate reconstructed from the $F_{S}$ series in L01. Solid curve denotes the reconstruction by Usoskin et al. (2002a). Solid circles correspond to the actual Climax NM count rates for 1951-2000. The hatched LIS area corresponds to an estimate of the local interstellar CR spectrum with uncertainties. Dashed L01 and dash-dotted S00, A00 lines represent the trend estimated for $\mathrm{L} 01$ reconstruction and the largest possible negative trend according to Ahluwalia (2000); Stozhkov et al. (2000), respectively.

the modulated spectrum $S$ and the specific yield function $Y$ (step 4)

$C R(N M)=\int_{P_{c}}^{\infty} S(P) \cdot Y(P) \cdot d P$,

where $P_{c}$ is the local rigidity cutoff and $P$ the CR particle's rigidity. Note that the integrand of Eq. (3) corresponds to the differential NM response function (Moraal et al., 2000). The CR intensity (in Climax NM count rates) so reconstructed is shown in Fig. 2 as the solid curve. In U02, we also reconstructed the ${ }^{10} \mathrm{Be}$ content in polar ice, and our results are in good agreement with both measured ${ }^{10} \mathrm{~B} e$ data (Beer et al., 1990; Bard et al., 1997) and calculations by McCracken and McDonald (2001).

\subsection{Comparison of the results}

Let us now analyse the two long-term CR intensity reconstructions depicted in Fig. 2 in more detail. Both depict clearly higher CR intensities around 1900 than nowadays. L01 estimated that "...the average fluxes of CR above $3 \mathrm{GeV}$ were approximately $15 \%$ higher in 1900 than they are now." On the other hand, the U02 CR reconstruction yields only a $7-8 \%$ increase in the Climax NM count rate around 1900. The long-term CR trend has been approximated in different ways. Note that the CR intensity around solar minima corresponds to a residual modulation in a quiet heliosphere. Stozhkov et al. (2000) calculated the trend in the residual 
modulation when trying to estimate the stability of CR intensity outside the heliosphere. They found that this trend is $-0.04 \pm 0.04 \% / y e a r$ for the Climax NM data after 1953 and attributed it to a possible supernova explosion in the vicinity of the solar system. This trend is depicted as a dashdotted line in Fig. 2. The CR data recorded by ion chambers since 1937 imply (Ahluwalia, 2000; Stozhkov et al., 2001; McCracken, 2001) that the CR trend was not steeper than the above estimate. The trend calculated similarly from the $\mathrm{CR}(\mathrm{NM})$ series reconstructed by L01, depicted as the dashed line in Fig. 2, is much steeper, about $-0.16 \pm 0.07 \% / y e a r$. The ratio of this trend and the trend estimated by Stozhkov et al. (2000) is about 4 (ranging roughly from 2 to 6 ). On the other hand, the trend calculated from the U02 CR(NM) series is only $-0.025 \pm 0.03 \% / y e a r$, in agreement with the results by Ahluwalia (2000); Stozhkov et al. (2000). Accordingly, since this result is based on the observed changes in solar activity, there is no need to introduce an additional effect by a supernova explosion, as suggested by Stozhkov et al. (2000).

An absolute upper bound for $\mathrm{CR}(\mathrm{NM})$ can be found from the local interstellar spectrum (LIS) of cosmic rays outside the heliospheric boundary (termination shock). It corresponds to the NM count rate if the Earth were located outside the heliosphere, i.e. the case of no heliospheric modulation, and can be calculated if the modulated CR spectrum in Eq. (3) is substituded by $S_{\text {LIS }}$. This upper bound was calculated by Usoskin et al. (2002b) with the LIS given by Burger et al. (2000) and the Climax NM specific yield function according to Debrunner et al. (1982) and Nagashima et al. (1989). We note that other estimates of LIS (see, e.g. Seo et al., 1994; Strong et al., 2000; Webber and Lockwood, 2001) are quite close to that given by Burger et al. (2000) above a few $\mathrm{GeV}$ energy range while they differ at lower energies. Also, there are some uncertainties related to the NM specific yield function (Clem and Dorman, 2000). Altogether, these uncertainties can lead to a few percent uncertainty in the calculated LIS, depicted by the hatched area in Fig. 2. (Possible large systematic errors are prevented by the overall normalization of the calculated NM count rates to the observed rates.) The exceedingly steep L01 trend makes the reconstructed CR intensity to reach and even exceed this upper bound during a few time intervals in the early part of the data interval. It has been suggested recently that the CR level might exceed LIS during extremely quite periods like the Maunder minimum (McCracken and McDonald, 2001). While there may be some modulation beyond the termination shock (McDonald et al., 2000), we consider here only the modulation inside the termination shock. Moreover, various indirect proxy data imply that the CR intensity was well below the LIS limit around 1900 (O'Brien et al., 1991; Bonino et al., 2001; McCracken and McDonald, 2001; Scherer et al., 2001). All these results are contrary to the steep trend obtained in L01 but are in accordance with the results presented in U02.

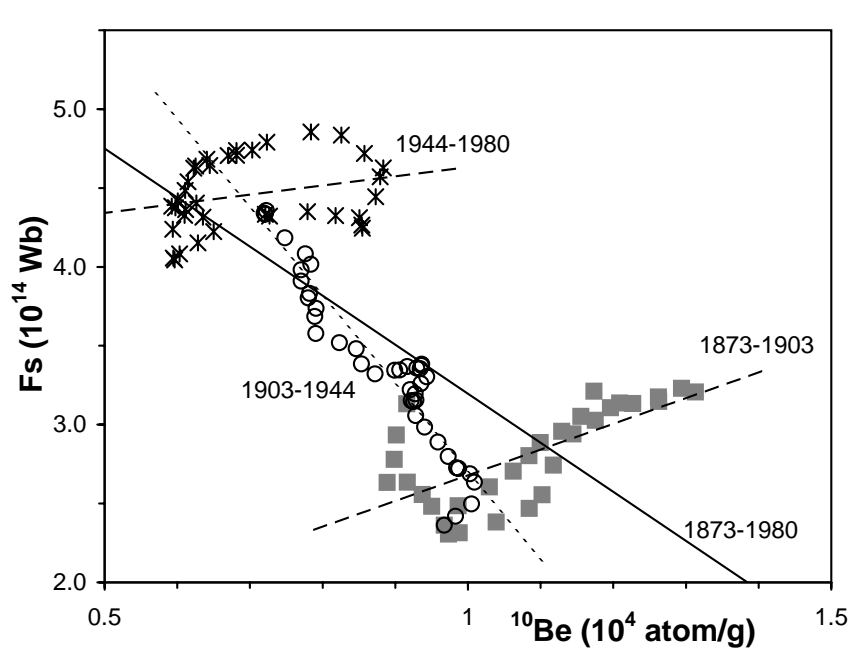

Fig. 3. Scatter plot of annual 11-year running mean values of $F_{S}$ vs. ${ }^{10} \mathrm{Be}$ concentration for $1873-1980$. Solid line represents the best linear regression for the entire period while squares and dashed line, open circles and dotted line, and asterisks and dashed line depict points and the best linear regression for 1873-1903, 1903-1944 and 1944-1980, respectively.

\section{$3 \quad F_{S}$ vs. cosmogenic ${ }^{10} \mathrm{Be}$ isotopes}

The linear relation between $F_{S}$ and $\mathrm{CR}(\mathrm{NM})$ was established in L01 during the last 50 years when the coronal source magnetic flux was much stronger than 130 years ago (Lockwood et al., 1999; Solanki et al., 2000). Therefore, Lockwood (2001) used the cosmogenic ${ }^{10} \mathrm{Be}$ isotope (Beer, 2000) as a proxy for long-term CR intensity in the past with the following linear relation between the annual 11-year running mean values of ${ }^{10} \mathrm{Be}$ and $F_{s}$ for the time interval $1873-1980$ :

$F_{S}=(6.3 \pm 0.3)-(3.1 \pm 0.4) \cdot C_{B e}$,

where $C_{B e}$ is given in $10^{4}$ atoms $/ \mathrm{g}$. We have used this linear relation to reconstruct $F_{S}$ from ${ }^{10} \mathrm{Be}$ data for the period of 1873-1980. The obtained $F_{S}$ series is depicted in Fig. 1. We note that this reconstruction is in good agreement with the results presented in L01. (see, e.g. the latter part of Fig. 14 there.)

One can see in Fig. 1 that, despite some similarity in the increasing trend, the source flux reconstructed from $C_{B e}$ (grey curve) is in disagreement with the flux derived from the $a a$ index (black curve). It is interesting to note that the relation between $F_{S}$ and $C_{B e}$ is strongly inhomogeneous. Figure 3 studies this relation by depicting the scatter plot separately for three periods. During periods 1873-1903 and 19441980 , when $F_{S}$ was roughly stable (see Fig. 1), the correlation between $F_{S}$ and $C_{B e}$ is slightly positive. (The slope of regression in Eq. (4) is $1.6 \pm 0.3$ and $0.5 \pm 0.4$, respectively.) This is also seen in Fig. 1 as a rough antiphase relation between the $F_{s}$ reconstructed from ${ }^{10} \mathrm{Be}$ and the "original" source flux. This may be related to an effect suggested by McCracken and McDonald (2001) that the ${ }^{10} \mathrm{Be}$ level can grow slowly 


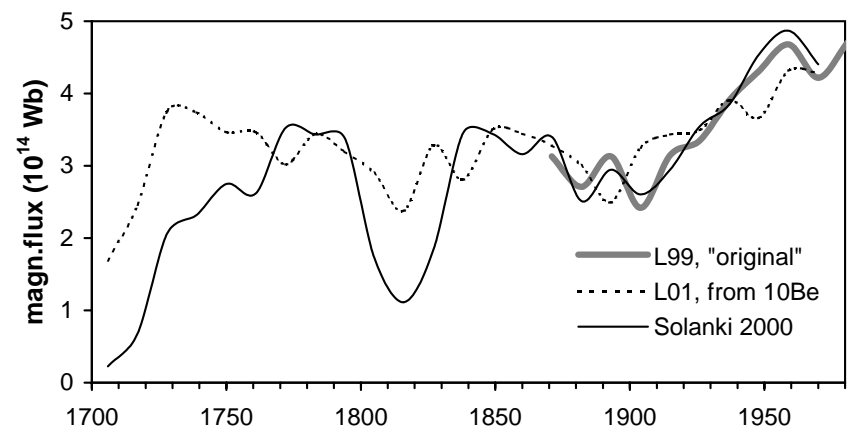

Fig. 4. 11-year running mean solar magnetic fluxes: the "original" coronal source flux $F_{S}$ by Lockwood et al. (1999), $F_{S}$ reconstructed by L01 from ${ }^{10} \mathrm{Be}$ series, and the open solar magnetic flux $F_{O}$ reconstructed by Solanki et al. (2000).

during constant solar activity times due to a replenishment of the slowly reacting heliosheath.

The two stable periods were intervened by a period of a fast monotonous increase of $F_{S}$ in 1903-1944. The correlation for that period is strongly negative, with a slope of $-5.5 \pm 0.3$. Accordingly, the relation between $F_{S}$ and $C_{B e}$ is completely different for stable periods and for periods of a fast monotonous change. Thus, no overall linear relation between $F_{S}$ and CR intensity exists, and the derived linear regression coefficients strongly depend on the chosen time interval. Therefore, the linear procedure of reconstructing $F_{S}$ from the ${ }^{10} \mathrm{Be}$ series, as suggested in L01, is questionable.

Recently, the open solar magnetic flux $F_{o}$, which is about twice $F_{s}$, was calculated by Solanki et al. (2000) for the period after Maunder minimum using sunspot number series (see Fig. 4). Although the (scaled) $F_{o}$ series is close to the "original" $F_{S}$ series by Lockwood et al. (1999) (correlation coefficient for 11-year averages is $r=0.98_{-0.05}^{+0.01}$ in 18681986); it is quite different from the $F_{S}$ series reconstructed from ${ }^{10} \mathrm{Be}\left(r=0.6_{-0.2}^{+0.1}\right.$ since 1700). As seen in Fig. 4, the difference between the magnetic flux reconstructions by L01 and by Solanki et al. (2000) is particularly large soon after Maunder minimum and during the Dalton minimum, i.e. when the solar activity has been exceptionally low. This is another demonstration of the problems which arise when linear relations are extrapolated beyond the range of values for which they were established.

\section{Concluding remarks}

We have shown that the long-term reconstruction of cosmic ray intensity for the last 130 years using a linear relation with the estimated solar coronal magnetic flux $F_{s}$ (Lockwood, 2001) leads to unphysical results. For example, the CR intensity in the Earth's vicinity is restricted to remain below an upper bound corresponding to the local interstellar spectrum, which is not obeyed within the framework of linear relations. This situation can be understood from two points of view. From a mathematical point of view, extrapolating a regres- sion far beyond the range where it has been established is not straightforward. In particular, the heliospheric CR modulation is very complicated and significantly nonlinear, and may be approximated by a linear regression only within a very limited interval. For example, the relation between $F_{S}$ and CR was experimentally established only for the last 45 years when $F_{S}$ was fairly stable and high. Then it was extended linearly by Lockwood (2001) for the last 130 years, over a time interval which includes periods of rapid changes of $F_{S}$ and periods of stable but much lower $F_{S}$ values than observed in recent times.

From a physical point of view, a linear influence of the coronal source flux (i.e. the intensity of the interplanetary magnetic field) on the cosmic ray intensity is oversimplified. While the interplanetary magnetic field is important for heliospheric CR modulation (Cane et al., 1999; Belov, 2000), the expected effect on cosmic rays is via the diffusion coefficient and is expected to be quite nonlinear. Moreover, the transport of cosmic rays in the heliosphere is also affected by other, non-negligible mechanisms like the heliospheric neutral sheet, interaction/rarefaction regions, drifts, IMF polarity, etc. (see, e.g. Potgieter et al., 1998; Belov, 2000, and references therein). These factors further strengthen the essentially nonlinear dependence of the long-term cosmic ray intensity on its main drivers, in particular the coronal source flux. We have demonstrated in this paper that the nonlinear dependence is already essential during the last 130 years, which invalidates the application of linear relations over this time interval. Therefore, the cosmic ray intensity as reconstructed by Lockwood (2001) after 1868 is not reliable. Since the nonlinear effects are even more important for still longer time intervals, the reconstruction of the very long-term coronal source flux after 1423 from the cosmogenic ${ }^{10} \mathrm{Be}$ isotopes is even more doubtful.

Acknowledgements. We thank the Academy of Finland for financial support. IGU acknowledges INTAS grant YSF 00-82. Chigaco University (NSF Grant ATM-9912341) and NOAA data center are acknowledged for Climax NM and $a a$ index data, respectively.

Topical Editor E. Antonucci thanks a referee for their help in evaluating this paper.

\section{References}

Ahluwalia, H. S.: On galactic cosmic ray flux decrease near solar minima and IMF intensity, Geophys. Res. Lett., 27, 1603-1606, 2000.

Bard, E., Raisbek, G. M., Yiou, F., and Jouzel, J.: Solar modulation of cosmogenic nuclide production over the last millenium: comparison between ${ }^{14} \mathrm{C}$ and ${ }^{10} \mathrm{Be}$ records, Earth and Planet. Sci. Lett., 150, 453-462, 1997.

Beer, J., Blinov, A., Bonani, G., et al.: Use of ${ }^{10} \mathrm{Be}$ in polar ice to trace the 11-year cycle of solar activity, Nature, 347, 164-166, 1990.

Beer, J.: Neutron monitor records in broader historical context, Space Sci. Rev., 93, 107-119, 2000.

Belov, A. V.: Large scale modulation: View from the Earth, Space Sci. Rev., 93, 79-105, 2000. 
Bonino, G., Cini Castagnoli, G., . Cane, D, Taricco, C., and Bhandari, N.: Solar modulation of the galactic cosmic ray spectra since the Maunder minimum, Proc. 27th Internat. Cosmic Ray Conf., Hamburg, 9, 3769-3762, 2001.

Burger, R. A., Potgieter, M. S., and Heber, B.: Rigidity dependence of cosmic ray proton latitudinal gradients measured by the Ulysses spacecraft: Implication for the diffusion tensor, J. Geophys. Res., 105, 27 447-27 455, 2000.

Cane, H. V., Wibberenz, G., Richardson, I. G., and von Rosenvinge, T. T.: Cosmic ray modulation and the solar magnetic field, Geophys. Res. Lett., 26, 565-568, 1999.

Clem, J. M. and Dorman, L. I.: Neutron monitor response functions, Space Sci. Rev., 93, 335-359, 2000.

Debrunner H., Flueckiger, E., and Lockwood, J. A.: Proc. 8th Europ. Cosmic Ray Symp., Rome, 1982.

Gleeson L. J. and Axford, W. I.: Solar Modulation of Galactic Cosmic Rays, Astrophys. J., 154, 1011-1026, 1968.

Lockwood, M.: Long-term variations in the magnetic field of the Sun and the heliosphere: the origin, effects and implications, J. Geophys. Res., 106 (A8), 16021-16038, 2001.

Lockwood M., Stamper, R., and Wild, M. N.: A doubling of the Sun's coronal magnetic field during the past 100 years, Nature, 399, 437-439, 1999.

Masarik, J. and Beer, J.: Simulation of particle fluxes and cosmogenic nuclide production in the Earth's atmosphere, J. Geophys. Res. 104 (D10), 12 099-12 111, 1999.

McCracken, K. G.: Variations in the production of ${ }^{10} \mathrm{Be}$ due to the 11 year modulation of the cosmic radiation, and variations in the vector geomagnetic dipole, Proc. $27^{\text {th }}$ Internat. Cosmic Ray Conf., Hamburg, 10, 4129-4132, 2001.

McCracken, K. G. and McDonald, F. B.: The long term modulation of the galactic cosmic radiation, 1500-2000, Proc. 27th Internat. Cosmic Ray Conf., Hamburg, 9, 3753-3756, 2001.

McDonald, F. B., Heikkila, B., Lal, N., and Stone, E. C.: The relative recovery of galactic and anomalous cosmic rays in the distant heliosphere: Evidence for modulation in the heliosheath, J. Geophys. Res., 105 (A1), 1-8, 2000.

Moraal, H., Belov, A., and Clem, J. M.: Design and co-ordination of multi-stational international neutron monitor network, Space Sci. Rev., 93, 285-303, 2000.

Nagashima K., Sakakibara, S., Murakami, K., and Morishita, I.: Response and yield functions of neutron monitor, Galactic cosmic ray spectrum and its solar modulation, derived from all the available world-wide surveys, Nuovo Cimento, 12 (C2), 173-209,
1989.

O'Brien, K., De La Zerda Lerner, A., Shea, M. A., and Smart, D. F.: The production of cosmogenic isotopes in the Earth's atmosphere and their inventories, in: The Sun in time, (Eds) Sonnet, C. P., Giampapa, M. S., and Matthews, M. S., the University of Arizona, Tucson, p. 317-343, 1991.

Potgieter, M. S.: The Modulation of Galactic Cosmic Rays in the Heliosphere: Theory and Models, Space Sci. Rev., 83, 147-158, 1998.

Scherer, K., Fichtner, H., Stawicki, O., and Fahr, H. J.: Cosmogenic element production in meteorites - the influence of long-term variation in heliospheric structure, Proc. 27th Internat. Cosmic Ray Conf., Hamburg, 10, 4031-4034, 2001.

Seo, E. S., McDonald, F. B., Lal, N., and Webber, W. R.: Study of cosmic-ray $\mathrm{H}$ and $\mathrm{He}$ isotopes at $23 \mathrm{AU}$, Astrophys. J., 432, 656-664, 1994.

Solanki, S. K., Schüssler, M., and Fligge, M.: Evolution of the Sun's large-scale magnetic field since the Maunder minimum, Nature, 408, 445-447, 2000.

Solanki, S. K., Schüssler, M., and Fligge, M.: Secular variation of the Sun's magnetic flux, Astron. Astrophys., 383, 706-712, 2002.

Stozhkov, Y. I., Pokrevsky, P. E., and Okhlopkov, V. P.: Long-term negative trend in cosmic ray flux, J. Geophys. Res., 105, 9-17, 2000.

Stozhkov, Y. I., Svirzhevsky, N. S., Makhmutov, V. S., et al.: Longterm cosmic ray observations in the atmosphere, Proc. 27th Internat. Cosmic Ray Conf., Hamburg, 9, 3883-3886, 2001.

Strong, A. W., Moskalenko, I. V., and Reimer, O.: Diffuse Continuum Gamma Rays from the Galaxy, Astrophys. J., 537, 763-784, 2000.

Usoskin, I. G., Mursula, K., Solanki, S., Schüssler, M., and Kovaltsov, G. A.: A physical reconstruction of cosmic ray intencity since 1610, J. Geophys. Res., 107 (A11), SSH 13-1-13-6, doi: 10.1029/2002JA009343, 2002a.

Usoskin, I. G., Alanko, K., Mursula, K., and Kovaltsov, G. A.: Heliospheric modulation strength during the neutron monitor era, Solar Phys., 207, 389-399, 2002b.

Webber, W. R. and Lockwood, J. A.: Voyager and Pioneer spacecraft measurements of cosmic ray intensities in the outer heliosphere: Toward a new paradigm for understanding the global solar modulation process: 1. Minimum solar modulation (1987 and 1997), J. Geophys. Res., 106 (A12), 29323-29332, 2001. 